\title{
A TECHNIQUE FOR HIGH-FREQUENCY SCANNING OF HIGH POWER LASER LIGHT FOR LASER-WIRE SCANNERS AT ELECTRON ACCELERATORS*
}

\author{
A. Bosco ${ }^{\#}$, G..A. Blair, S. T. Boogert, G. Boorman, \\ John Adams Institute at Royal Holloway University of London, Egham, UK
}

\begin{abstract}
Electro-optic techniques might allow implementing a laserwire scanner for intra-train scanning at the ILC with scanning speed in excess of $100 \mathrm{kHz}$. A scanner capable of running at such a rate would in fact provide information about the particle beam size in about one hundred different positions along the bunch train (approximately 1ms long for the ILC). The design of an electro-optic deflector capable to scan within 10-100 microsecond is presented, discussed and analytically treated.
\end{abstract}

\section{INTRODUCTION}

Laser-wires have been proposed in order to measure the properties of high energy electron beams, in particular the ILC [1], where electron and positron beams with energy up to $500 \mathrm{GeV}$ will be collided and where the final focus spot-size will require demagnification of the vertical dimension to approximately $5 \mathrm{~nm}$. Some of the challenges posed by the ILC on the upstream laser-wire systems have been discussed [2]. In particular, it will be necessary to focus intense beams of laser-light down to micron scales and, in order to scan the electron beams within a single pulse (or train) of bunches, it will be necessary to scan at very high frequencies, in excess of 10-100 $\mathrm{kHz}$. It has been proposed [3] to use electro-optic (EO) techniques for this purpose, exploiting the refraction at the output face of a prism made of EO materials; in this paper a different configuration of EO high-frequency driver is explored in order to obtain more efficient deflection strength.

\section{PRINCIPLE OF OPERATION}

The principle of operation of the presented device is based upon the generation of a linear gradient of electrostatic field across the transversal laser beam crosssection.

In fact, through the EO effect, an EO material experiences a change $\Delta \mathrm{n}$ in the refractive index $\mathrm{n}_{0}$ given by [4]:

$$
\Delta n=\frac{1}{2} n_{0}^{3} r_{33} E_{z}
$$

where $r_{33}$ is the EO coefficient which couples the electrostatic field along the crystallographic c-axis and a parallel polarized light [note that the more general coupling

*Work supported by the Royal Society, PPARC LC-ABD collaboration and the Commission of the European Communities under the 6th Framework Programme Structuring the European Research Area, contract nr RIDS-RIDS-011899.

\# alessio.bosco@rhul.ac.uk

06 Instrumentation, Controls, Feedback \& Operational Aspects formula is tensorial and eq. (1) is valid only for a particular class of materials such as the one treated in the text]. Now, if all along the EO crystal length an electrostatic field with linear transversal gradient is applied, the right side of the laser beam will travel at a different speed than the left one (the refractive index being different) This will result in a deflection of the wave-front directly proportional to the refractive index difference and the propagation length. The advantage of this configuration is obvious: the deflection obtainable increases by increasing the crystal length.

In order to obtain a linear gradient of the electro-static field, we propose to use EO crystals within an electrostatic quadrupole configuration as shown in fig. 1 While such devices are commercially available for low power applications, there are many challenges to obtain a large aperture device which would allow high-power laser use and optical quality sufficient for use within a laser-wire system (the largest clear aperture available in the market is around $3 \mathrm{~mm}$ diameter).

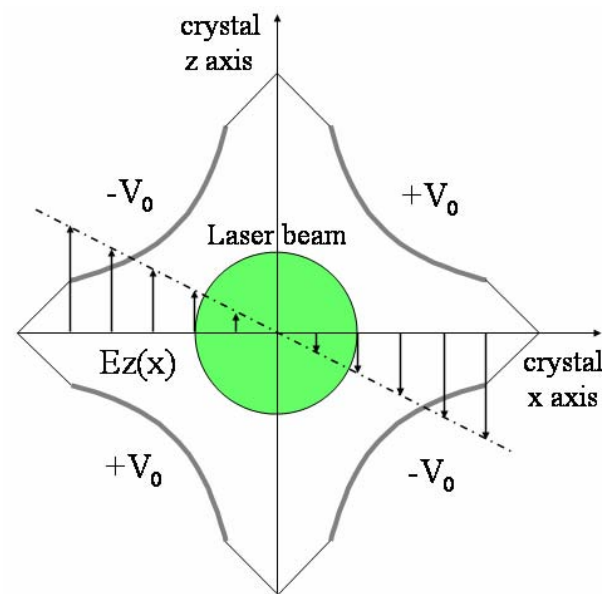

Figure 1: Schematic picture of a quadrupole EO deflector. The value of the refractive index changes from left to right by $2 \Delta \mathrm{n}$ given by Eq. $1\left(\mathrm{E}_{\mathrm{z}}\right.$ is the value assumed at the edges of the beam). After propagating by a distance $L$ through the crystal the left side will have travelled by a distance $\Delta \mathrm{L}=2 \Delta \mathrm{n} / \mathrm{n}_{0}$ shorter. This will result to a deflection of the wavefront $\theta=\Delta \mathrm{L} / 2 \mathrm{w}$ (where $2 \mathrm{w}$ is the beam diameter).

\section{DESIGN DETAILS}

The problem of obtaining a large aperture EO scanner is fundamental. In fact, if an EO device with large clear aperture obtained with an EO crystal shaped as in fig. 1 
would require growing it with extremely large dimensions $\left(16 \times 16 \mathrm{~mm}^{2}\right.$ for a clear aperture of $\left.6 \mathrm{~mm}\right)$ with obvious technical growth problems.

We propose a hybrid solution in order to overtake this problem. In fig. $2 \mathrm{a}$ is depicted a detailed drawing of a scanner with a clear aperture of $8.5 \mathrm{~mm}$, capable to accept a beam diameter up to $7 \mathrm{~mm}$ (within the diffraction limit $8.5 \mathrm{~mm} / 1.22$ [4]).

Using the dimensions as in fig 2a, we realized a prototype with hyperbolic electrodes and a $50 \mathrm{~mm}$ long crystal of LNB (being grown at the time of writing). In fig. $2 b$ is reported a picture of the realized crystal holder with hyperbolic electrodes.

Furthermore, the hybrid solution we present is extremely flexible, it indeed allows us to change the electrode shape just by producing a differently shaped
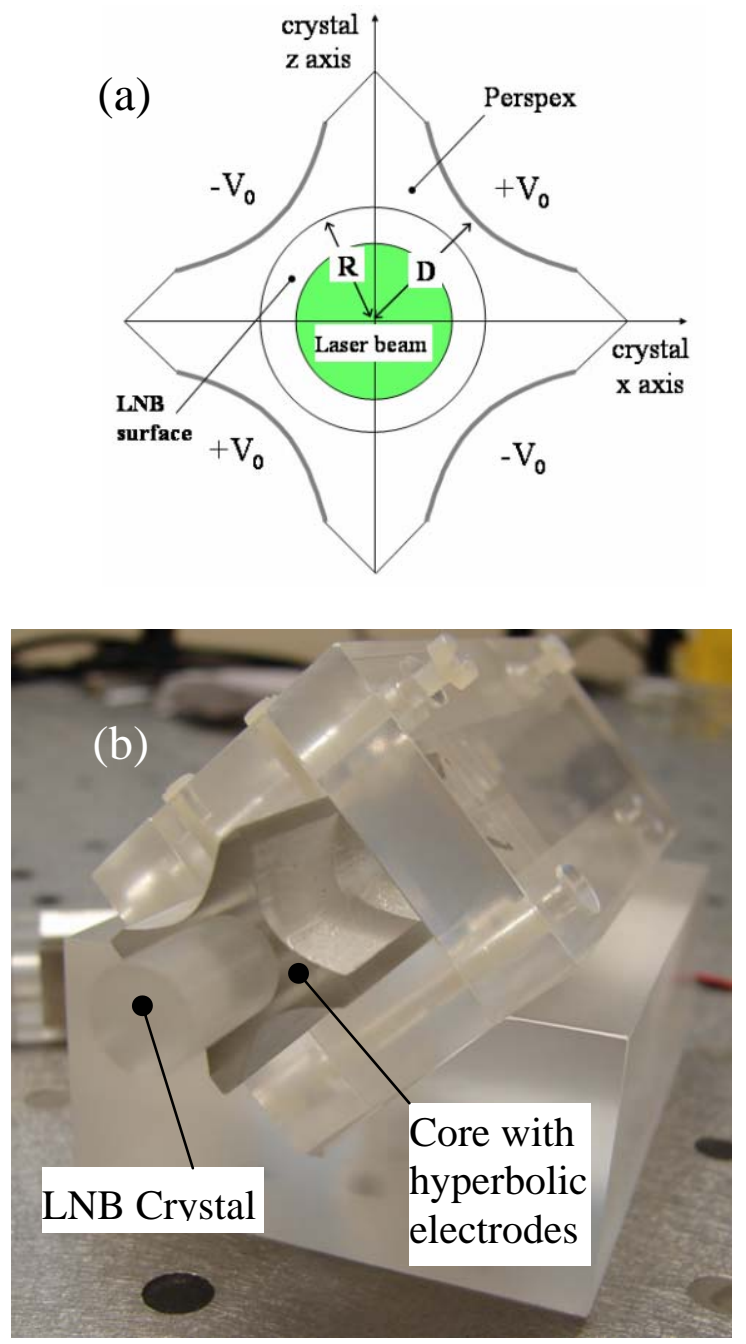

Figure 2: (a) Drawing of the designed hybrid structure. $\mathrm{R}$ is the LNB radius ( $8.5 \mathrm{~mm})$, D the inner (minimum) distance between the centre and the hyperbolic electrodes. The square face of the perspex holder is $16 \times 16 \mathrm{~mm}^{2}$. (b) Picture of the realized crystal holder with hyperbolic shaped electrodes. holder (made in our facilities using inexpensive materials), in order to optimize the distribution of the electro-static field within the EO material. In fact, in the following an analytical treatment of the problem is presented and a numerical optimization of the electrodes' shape and relevant dimensions obtained.

\section{ANALYTICAL TREATMENT}

If the electric field is made up of a dominant linearly dependent (quadrupole) component $x d E_{z} / d x$ plus an additional component $\delta E_{z}$ due to the presence of higherorder multipoles, so that $E_{z}=x d E_{z} / d x+\delta E_{z}$. The effect will be a rotation $\Delta \theta$ of the wavefront after transmission through a length $L$ of crystal is given by:

$$
\Delta \theta=\frac{d n}{d x} L=\frac{1}{2} n^{3} r_{33} L \frac{d E_{z}}{d x}
$$

plus a residual distortion proportional to the term $\delta E_{z}$ which is currently being calculated and minimized..

The EO crystal we consider (LNB) has a non-isotropic permittivity, characterized here by two values $\varepsilon_{33}$ along the electro-optic axis (the vertical axis in Fig1) and $\varepsilon_{11}$ along the perpendicular axis.

In the following we define $\varepsilon_{33}=\left(\varepsilon_{11}+\varepsilon_{33}\right) / 2$ and $\Delta \varepsilon_{1}=$ $\left(\varepsilon_{11}-\varepsilon_{33}\right) / 2$. We also introduce dimensionless variables $\rho=$ $r / D, \eta=R / D$, where $r$ is the radial variable, $D$ the inner (minimum) distance between the centre of the device and the high-voltage electrode with applied electric potential $V_{0}$, and $R$ is the radius of the crystal as illustrated in Fig. 2a.

The outer material has relative permittivity $\varepsilon_{2}$. In general, when $\rho \leq \eta$ for a perfect quadrupole field $V=V_{2} \rho^{2} \sin 2 \theta$ and for $\rho>\eta$ :

$$
\begin{gathered}
\frac{V}{V_{2}}=\frac{1}{2}\left[\left(1+\frac{\varepsilon_{1}}{\varepsilon_{2}}\right) \rho^{2}+\left(1-\frac{\varepsilon_{1}}{\varepsilon_{2}}\right) \frac{\eta^{4}}{\rho^{2}}\right] \sin 2 \theta+ \\
+\frac{1}{8} \frac{\Delta \varepsilon_{1}}{\varepsilon_{2}}\left[\frac{\rho^{4}}{\eta^{2}}-\frac{\eta^{8}}{\rho^{4}}\right] \sin 4 \theta .
\end{gathered}
$$

The gradient of the $y$-component of the electric field $E_{z}$ within the crystal is then:

$$
\frac{d E_{z}}{d x}=\frac{2 V_{2}}{D_{i}^{2}}
$$

The relationship between applied voltage $V_{0}$ and the parameter $V_{2}$ is given by:

$$
\frac{V_{0}}{V_{2}}=\frac{1}{2}\left[\left(1+\frac{\varepsilon_{1}}{\varepsilon_{2}}\right) \rho^{2}+\left(1-\frac{\varepsilon_{1}}{\varepsilon_{2}}\right) \eta^{4}\right] .
$$


Ideally $\eta$ should be as close to 1 as possible in order to maximize the electric field within the crystal; for the parameters listed in Tab.1, $V_{0} / V_{2}=4.8$.

The shape of the electrode that is needed to obtain a quadrupole field within the crystal can be obtained by imposing $V(\rho \theta)=V_{0}$ in Eq.3; the resulting electric equipotential lines and electrode optimized shape are shown in fig.3. The exact shape and dimensions of the system needs to be optimized in order to improve the linearity of the fields within the crystal; alternative configurations of electrodes are currently under study.

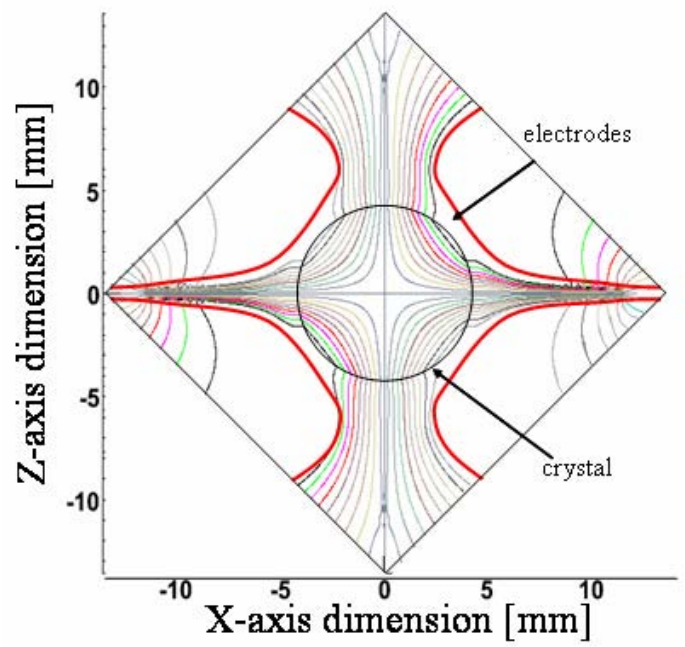

Figure 3: Plot of the equipotential lines generated by the shaped electrodes. The shape of the electrodes was calculated by imposing the lines to be hyperbolic within the EO crystal.

Table 1. List of parameters used for the design

\begin{tabular}{|c|c|c|c|}
\hline Parameter & Symbol & Value & Unit \\
\hline Inner electrode dim. & $D$ & 5 & $\mathrm{~mm}$ \\
\hline Outer relative perm. & $\varepsilon_{2}$ & 3.4 & \\
\hline Crystal rel. perm. $x$ & $\varepsilon_{11}$ & 85 & \\
\hline Crystal rel. perm. $z$ & $\varepsilon_{33}$ & 29 & \\
\hline Crystal refr.. index & $\mathrm{n}_{03}$ & 2.25 & \\
\hline Crystal EO coeff. & $\mathrm{r}_{33}$ & 31 & $\mathrm{pm} / \mathrm{V}$ \\
\hline Crystal length & $L$ & 50 & $\mathrm{~mm}$ \\
\hline Deflection Strength & $\Delta \theta /\left(V_{0} L\right)$ & 2.9 & $\mu \mathrm{rad} / \mathrm{kV} / \mathrm{mm}$ \\
\hline
\end{tabular}

\section{POSSIBLE FAST SCANNER SCHEME}

Here we will briefly describe how the EO deflector presented above could be implemented into a LW scanner capable to perform a scan within $77 \mu$ s.

A mode-locked laser with synchronizing electronics (Time-Bandwidth CLX1100) runs at $130 \mathrm{kHz}(7.7 \mu \mathrm{s}$ period) delivering a pulse $12 \mathrm{ps}$ long. An amplifier, with a gain of $60 \mathrm{~dB}(1000 \mathrm{X})$ and bandwidth of $75 \mathrm{kHz}$, is fed by a stepped ramp function from 0 to $5 \mathrm{~V}$ to generate the $\mathrm{HV}$ ramp (from 0 to $5 \mathrm{kV}$ ) for driving the EO scanner.

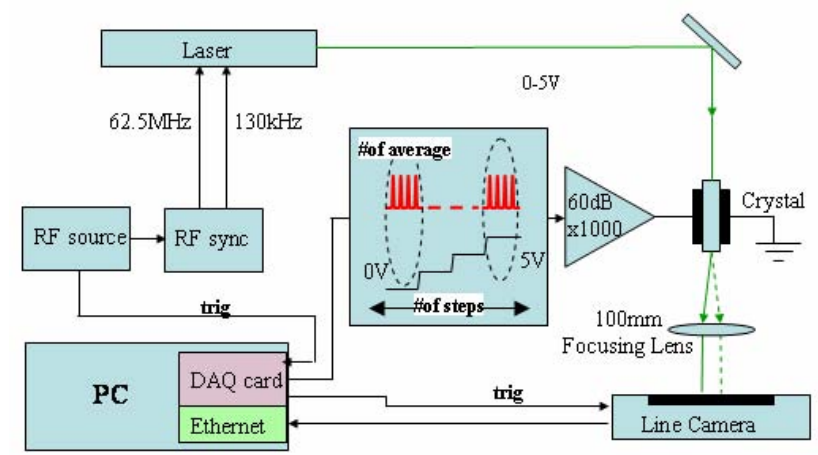

Figure 4: Schematic diagram of the experimental set-up being set up at RHUL.

The scanner has the dimensions as in Tab.1. By applying a ramp from 0 to $5 \mathrm{kV}$ we expect a deflection of $0.75 \mathrm{mrad}$ $\left(\left(2.9 \mu \mathrm{rad} \cdot \mathrm{V}^{-1} \cdot \mathrm{mm}^{-1} \times 5 \mathrm{kV} \times 50 \mathrm{~mm}\right)\right)$. Focusing this beam with a $100 \mathrm{~mm}$ lens we can obtain a beam waist of $5 \mu \mathrm{m}(\sigma=2.5 \mu \mathrm{m})$ and a scan range of $75 \mu \mathrm{m}$.

For measuring the profile and the position of every second pulse of the scanned laser beam, we will use a CCD line camera, consisting of a single row of 1024 pixels, can be read out via Ethernet at a rate in excess of $68 \mathrm{kHz}$.

The large bandwidth of the HV amplifier will allow amplifying a step function of 10 steps around $8 \mu$ s each, in a way to scan ten laser pulses in ten different positions within $80 \mu \mathrm{s}$. In case an average over multiple interaction has to be done, each step will be as long as a multiple of the laser pulse spacing $(7.7 \mu \mathrm{s})$.

\section{CONCLUSION}

In conclusion, we proposed a hybrid solution for an EO scanner with large aperture capable of operating with high laser power. The calculated deflection is expected to be $0.75 \mathrm{mrad}$ for a laser beam diameter of $7 \mathrm{~mm}$. The expected $\sigma$ of $2.5 \mu \mathrm{m}$ and scan range of $75 \mu \mathrm{m}$ makes it suitable for a fast laserwire scanner.

Experimental test on the realized prototype are being performed using a laser at $130 \mathrm{kHz}$ and a $\mathrm{HV}$ ramp as short as $80 \mu \mathrm{s}$.

Furthermore, the presented analytical model will enable us to optimize the electrode shape for more efficient performances.

\section{REFERENCES}

[1] ILC Baseline Conceptual Design (2006): http://www.linearcollider.org/.

[2] Agapov et al., Submitted to Phys. Rev. ST-AB.

[3] Bosco et al., Proceedings of EPAC06 (2006).

[4] Yariv, Pochi, Yeh, "Optical waves in crystals: propagation and control of laser radiation” John Wiley and Sons (2002). 